\title{
Diseño, aplicación y efecto de un programa para la mejora de la creatividad en Educación Infantil.
}

(Design, implementation and impact of a creativity enhancement program in Preschool Education.)

\author{
María del Carmen Castillo Cobo \\ Universidad de Granada, España. \\ María Jesús Caurcel Cara \\ Facultad de Ciencias de la Educación, Universidad de Granada, España. \\ Helena Chacón López \\ Facultad de Ciencias de la Educación, Universidad de Granada, España.
}

Fecha recepción: 28-05-2018

Páginas 01-10

Fecha aceptación: 30-06-2018

\section{Resumen.}

Esta investigación presenta el diseño, aplicación y evaluación de un programa de intervención para la mejora y potenciación de la creatividad en Educación Infantil. Objetivo: determinar la eficacia del programa "¿Te atreves a crear?". Método: participaron 27 alumnos de un aula de 4 años. Para evaluar al alumnado y determinar el impacto del programa se ha utilizado el test de evaluación de la creatividad de Torrance, con un diseño cuasi-experimental pretest-postest; un registro anecdótico y unos cuestionarios de evaluación y autoevaluación del programa. Resultados: los resultados han demostrado que el programa contribuye a la mejora de la creatividad, y que el tamaño del efecto ha sido grande. Conclusiones: es posible estimular el potencial creativo de los niños, y esto va a tener una influencia positiva sobre su desarrollo posterior, por lo que la escuela debe adoptar enfoques educativos que permitan impulsar las competencias creativas de los estudiantes.

Palabras-clave: creatividad; infancia; programa, evaluación; diseño

\begin{abstract}
.
This research includes the design, application and evaluation of an intervention program for the improvement and enhancement of creativity in students of Pre-school Education. Objectives: to determine the effectiveness of the program " $i T e$ atreves a crear?". Method: 27 students in a classroom of 4 years old participated in the study. To evaluate the students and to know the impact of the program the Torrance Test of Creativity Thinking was used, with a quasi-experimental design pretest-posttest; as well as a register of anecdotes and evaluation and self-evaluation questionnaires of the program. Results: the results showed that the program has contributed to the improvement of the creativity and the size of the effect has been great. Conclusions: it is possible to stimulate the creative potential of children, and this will have a positive
\end{abstract}


influence on his later development, so schools should addopt educational approaches that will inspire the creative skills of the students.

Keywords: creativity; childhood; program, evaluation; design

\section{1.-Introducción.}

Según Marín-lbáñez y Torre (1991), los componentes de la creatividad son: fluidez (cantidad de ideas y respuestas que una persona puede producir respecto a un tema 0 problema determinado); flexibilidad (variedad y heterogeneidad de las ideas producidas); originalidad (rareza relativa de las ideas producidas); y, elaboración (precisión y meticulosidad con que ha sido realizada una obra). Estos cuatro factores, se convierten en los criterios que permiten evaluarla (Khatena y Torrance, 1976, citado en López-Megías y Fernández-Castillo, 2018).

La creatividad, como señala Cemedes (2008) es una característica inherente al ser humano; pues existe el impulso de experimentar, explorar, investigar, relacionar, en definitiva, de crear. Además, es una capacidad que puede ser cultivada y estimulada por el entorno en el que se desarrolla el niño (Cemedes, 2008; López-Megías y Fernández-Castillo, 2018).

Algunos autores (Gardner, 1982 citado en Alfonso-Benlliure, Meléndez y GarcíaBallesteros, 2013), describen los años preescolares como una edad de oro de la creatividad. En estos años, el niño realiza el mayor desarrollo neuronal en el ser humano (Dacey, 1989 citado en Cemedes, 2008), desea resolver problemas por sí mismo, enfrentarse a situaciones nuevas y buscar soluciones; es decir, se encuentra en una etapa ideal para el desarrollo de su creatividad (Cemedes, 2008), sin embargo, si en este período se coarta su naturalidad, su libertad, no se refuerza la seguridad en sí mismo y se le presiona con actitudes autoritarias y rigidez, el pensamiento creativo decrece. En este mismo sentido, Gardner (1982 citado en Alfonso-Benlliure et al., 2013), considera que los niños de Educación Infantil tienen altos niveles de capacidad creativa, pero cuando pasan a niveles superiores en el sistema educativo, su creatividad tiende a disminuir a medida que aprenden conformidad. Urban (1991 citado en Alfoso-Benlliure et al., 2013), también, encuentra una disminución de la creatividad de los niños al comienzo de la Educación Primaria, mostrando que su pensamiento se vuelve más lógico y convencional, ajustándose a las demandas escolares.

Educar la creatividad en los escolares, con el fin de que sean capaces de descubrir y desarrollar su potencial creativo, a través de los distintos canales de expresión de que dispone el ser humano, parece haberse convertido en uno de los grandes retos de la educación (Fernández y Peralta, 1998). Así, como señalan Muñoz (1994) y Torre (1995), el objetivo, es promover la enseñanza creativa, a través de una mayor implicación por parte del docentes y de la adquisición de destrezas y/o técnicas creativas por parte del alumnado.

Generalmente, los enfoques educativos convencionales han tratado de desarrollar el pensamiento convergente, pero los enfoques actuales se proponen impulsar las 
capacidades creativas y el pensamiento divergente en los individuos (Beltrán, Garzón y Burgos, 2016 citado en López-Megías y Fernández-Castillo, 2018; Yildirim, 2010, López-Megías y Fernández-Castillo, 2018). Pero, cualquier metodología, como señala Cemades (2008), va a depender en gran medida del profesor, de su ideología, formación, capacidad y características propias. El docente, debe creer lo que está haciendo, debe ser reflexivo, y abierto a los cambios necesarios que le permitan obtener los resultados deseados. Y para ello, debe formarse y aprender a observar el proceso que se lleva a cabo en su aula.

Existen diferentes programas diseñados para estimular la creatividad, que difieren en el enfoque y el contenido, en función del modelo de creatividad subyacente y de la experiencia acumulada de la evaluación de estos programas (Fernández y Peralta, 1998).

Según la revisión realizada por Dziedziewicz, Oledzka y Karwowski (2013), los programas que estimulan la creatividad infantil se basan en actividades de lenguaje, música, movimiento y dramatización, diseñadas para ser trabajadas principalmente en grupo. Algunos programas, como el Garaigordobil (2006), se basan en la estimulación polisensorial, con un fuerte peso de las actividades artísticas creativas.

Diferentes metaanálisis (Ma, 2006; Scott, Leritz y Mumford, 2004a, 2004b) han confirmado la eficacia de los programas orientados a promover la creatividad en los niños de Educación Infantil (Antonietti, 2000; Castillo, 1998; Cliatt, Shaw y Sherwood, 1980; Mohanty y Hejmadi, 1992; Prieto, López, Bermejo, Renzulli y Castejón, 2002; Useem y Downie, 1976). Programas más recientes, como el de Dziedziewicz et al. (2013) o el de Alfonso-Benlliure et al. (2013), también han demostrado su efectividad para el desarrollo de la capacidad creativa en edades tempranas. Además, se ha encontrado un efecto moderador de la edad, es decir, han sido más eficaces con los participantes de menor edad, lo que resalta la importancia de este tipo de trabajos durante la Educación Infantil (Garaigordobil y Berrueco, 2011) y el porqué de este programa.

\section{1.-Presentación del programa “¿Te atreves a crear?”.}

El programa "¿Te atreves a crear?" consta de un total de 13 sesiones diarias (ver Tabla 1), con una duración de entre una hora o una hora y media. Las sesiones se organizan manera que cada día se trabajen diferentes tipos de contenidos: pensamiento divergente, expresión plástica, musical y emocional. El programa pretende el desarrollo de las habilidades creativas a través de diferentes juegos y actividades lúdicas, de forma colectiva, con todo el grupo-clase, en su mayor parte, y en pequeños grupos, en algunas de sus actividades, favoreciendo el trabajo en grupo y cooperativo. 
Tabla 1: Temporalización del programa.

\begin{tabular}{|c|c|c|c|c|}
\hline Sesión & Nombre & Contenido & Actividades & Duración \\
\hline 1 & ¡Vamos a ser pintores! & $\begin{array}{l}\text { Pensamiento } \\
\text { divergente } \\
\text { Expresión emocional }\end{array}$ & $\begin{array}{l}\text { ¿Qué vemos? } \\
\text { Diario del día }\end{array}$ & $30^{\prime}$ \\
\hline 2 & $\begin{array}{l}\text { ¡Nunca nos aburrimos } \\
\text { y siempre bailamos! }\end{array}$ & $\begin{array}{l}\text { Expresión emocional } \\
\text { Pensamiento } \\
\text { divergente } \\
\text { Expresión musical }\end{array}$ & $\begin{array}{l}\text { Diario del día } \\
\text { Esto era muy } \\
\text { aburrido, ahora es... } \\
\text { ¡A bailar! }\end{array}$ & $60^{\circ}$ \\
\hline 3 & ¡Construimos! & $\begin{array}{l}\text { Expresión emocional } \\
\text { Expresión plástica }\end{array}$ & $\begin{array}{l}\text { Diario del día } \\
\text { Hacemos figuras con } \\
\text { el tangram } \\
\text { Acabamos el dibujo }\end{array}$ & $60^{\circ}$ \\
\hline 4 & $\begin{array}{l}\text { ¡Primero imaginamos y } \\
\text { después nos } \\
\text { ensuciamos! }\end{array}$ & $\begin{array}{l}\text { Expresión emocional } \\
\text { Pensamiento } \\
\text { divergente } \\
\text { Expresión plástica }\end{array}$ & $\begin{array}{l}\text { Diario del día. } \\
\text { Relax imaginativo } \\
\text { Pintamos con } \\
\text { nuestras manos }\end{array}$ & $60^{\circ}$ \\
\hline 5 & ¡Creadores en acción! & $\begin{array}{l}\text { Expresión emocional } \\
\text { Expresión plástica }\end{array}$ & $\begin{array}{l}\text { Diario del día } \\
\text { Nuestra primera obra } \\
\text { Nuestro cuento }\end{array}$ & $80^{\circ}$ \\
\hline 6 & $\begin{array}{l}\text { ¡Nos aburría lo de } \\
\text { siempre! }\end{array}$ & $\begin{array}{l}\text { Expresión emocional } \\
\text { Expresión plástica }\end{array}$ & $\begin{array}{l}\text { Diario del día } \\
\text { Damos color y forma }\end{array}$ & $60^{\circ}$ \\
\hline 7 & ¡Locos pero divertidos! & $\begin{array}{l}\text { Expresión emocional } \\
\text { Expresión musical } \\
\text { Pensamiento } \\
\text { divergente }\end{array}$ & $\begin{array}{l}\text { Diario del día } \\
\text { ¡A bailar! } \\
\text { Ideas locas }\end{array}$ & $70^{\circ}$ \\
\hline 8 & ¡Qué feo! & $\begin{array}{l}\text { Expresión emocional } \\
\text { Pensamiento } \\
\text { divergente } \\
\text { Expresión plástica }\end{array}$ & $\begin{array}{l}\text { Diario del día } \\
\text { ¿Qué pirata más feo! } \\
\text { Nuestro cuento }\end{array}$ & $80^{\circ}$ \\
\hline 9 & ¡La clase de mimos! & $\begin{array}{l}\text { Expresión emocional } \\
\text { Expresión corporal } \\
\text { Expresión plástica }\end{array}$ & $\begin{array}{l}\text { Diario del día } \\
\text { ¡Somos mimos! } \\
\text { Nuestro cuento }\end{array}$ & $80^{\circ}$ \\
\hline 10 & Ku- ku, ku-ku... & $\begin{array}{l}\text { Expresión emocional } \\
\text { Expresión plástica }\end{array}$ & $\begin{array}{l}\text { Diario del día } \\
\text { ¡El pájaro sin plumas! }\end{array}$ & $50^{\circ}$ \\
\hline
\end{tabular}

Fuente: elaboración propia (2018)

\section{2.-Objetivo.}

Este estudio pretende determinar la eficacia de un programa ad hoc para el desarrollo de la creatividad del grupo al que se dirige. 


\section{3.-Metodología.}

Atendiendo al objetivo de esta investigación se ha seguido un diseño cuasiexperimental, pretest-postest, de grupo único. En el centro escolar, existe una sola línea en la etapa de Educación Infantil, por lo que, no se ha podido contar con grupo control..

\section{1.-Participantes.}

El programa se implementa en el aula de 4 años de Educación Infantil, de una escuela privada-concertada de la ciudad de Granada (España). Se ha utilizado un muestreo probabilístico de conveniencia, participando 27 alumnos (15 niños y 12 niñas, $\bar{x}=1.44, D T=.51$ ), con una media de edad de 4.84 años $(D T=.27)$. En cuanto a sus características como grupo, antes del inicio del programa, destaca su escasa colaboración grupal y su falta de respeto hacia los compañeros. Los niños, en general tienen una capacidad intelectual notable, con conocimientos avanzados en lecto-escritura, grafomotricidad y matemáticas; en cambio, tienen olvidada una parte muy importante de su educación, la creatividad.

\section{2.-Instrumentos.}

El instrumento seleccionado ha sido la edición en español del test de Torrance, (Test of Creative Thinking) (Torrance, Ball y Safter, 1992), en la forma gráfica A: Thinking creatively with pictures; pues es uno de los pocos test que se pueden aplicar a casi cualquier edad. En esta forma, se pide al niño que realice una serie de dibujos diferentes y le dé un título interesante. En la actividad 1, denominada Construcción de dibujos, el niño debe realizar un dibujo a partir de una forma curvada. En la actividad 2, Terminación de dibujos, se trata de completar las figuras añadiendo unas líneas. Y, por último, la actividad 3, Líneas, consiste en realizar dibujos diferentes, a partir de pares de líneas verticales, en 10 minutos.

El test mide los 4 componentes de la creatividad: fluidez (número de ideas emitidas), flexibilidad (variedad de ideas emitidas en función del número de categorías existentes), originalidad (novedad de las ideas en función de su frecuencia) y elaboración (detalles que mejoran la calidad de las ideas emitidas). También, se puntúan con "bonus" otros 13 aspectos diferentes de cada uno de los dibujos (si tiene humor, utiliza colores, tiene fantasía, movimientos en acción, etc.). Para la puntuación de los mismos, se ha utilizado acuerdo inter-jueces.

Además, para evaluar el proceso de implementación, se ha realizado un registro anecdótico tras cada sesión, anotando el devenir, las dificultades surgidas y el progreso del alumnado, así como cualquier otro aspecto destacable. Los participantes, también, evalúan las sesiones en una asamblea donde todos pueden opinar, que también queda reflejado en el registro anecdótico. Igualmente, el 
programa, ha sido evaluado en sí mismo, a través de dos cuestionarios, cumplimentados por la tutora del grupo-aula y la persona encargada de la implementación.

\section{3.-Procedimiento.}

Para llevar a cabo el estudio durante el segundo y tercer trimestre del curso académico 2015-2016 (ver Tabla 2), se solicitó permiso al equipo directivo del centro escolar y al tutor de grupo; así como a los familiares o tutores a través de una nota informativa, donde se presentaba la investigación, se explicaban los objetivos y se aclaraba que podían rehusar la participación del menor en el mismo. En ningún caso se plantearon problemas y todos los familiares dieron su consentimiento.

Tabla 2: Pasos para la implementación y evaluación del programa

\begin{tabular}{ll}
\multicolumn{1}{c}{ TAREA } & \multicolumn{1}{c}{ FECHA } \\
\hline Solicitud de permisos & 1-20 de marzo \\
Pretest & 16-27 de marzo \\
Implementación del programa & 7 de abril-19 de mayo \\
Postest & $20-29$ de mayo \\
Evaluación del programa & 8-12 de junio \\
\hline
\end{tabular}

Fuente: elaboración propia (2018)

Antes de comenzar la recogida de datos, se estableció un periodo de dos semanas, en el que los niños pudieran familiarizarse con la persona que iba a llevar a cabo la recogida de datos. Después, se procedió al establecimiento de la línea base con la pasación del test de Torrance (Torrance, Ball y Safter, 1992) (pretest), -durante dos semanas-, la puntuación por cada una de las jueces y el análisis de resultados, de tal manera que el día 7 de abril, se estaba en disposición de comenzar con la implementación del programa.

Se empezaron a trabajar, diariamente, las sesiones propuestas con los alumnos, durante un periodo de ocho semanas del tercer trimestre. El primer día, se les explicó que a partir de ese momento se iban a convertir en pintores, en bailarines, en mimos, etc.; para que todo lo planteado fuese un juego divertido para ellos, y pudiesen meterse fácilmente en el papel.

Para evaluar sí se había producido alguna mejoría en su creatividad, se volvió a pasar el test (postest). Y, finalmente, se procedió a la evaluación del programa en sí mismo. 


\section{4.-Análisis de datos.}

Los datos recogidos fueron tratados con el paquete estadístico SPSS versión 22.0 para Windows. Para establecer la normalidad de la muestra se utilizó la prueba Shapiro-Wilk, aceptándose la hipótesis nula, es decir, que la distribución muestra es normal. Para conocer la distribución de los resultados se calcularon los estadísticos descriptivos (media y desviación típica). En relación con el efecto del programa, se compararon los valores pretest-postest a través de la prueba t para muestras relacionadas y se calculó el tamaño del efecto utilizando el parámetro de de Cohen (1988).

\section{4.-Discusión de los resultados.}

Se ha utilizado una doble vía para determinar la eficacia de la intervención (ver Tabla $3)$.

Tabla 3: Efecto del programa

\begin{tabular}{|c|c|c|c|c|c|c|c|}
\hline \multirow{2}{*}{ CREATIVIDAD } & \multicolumn{2}{|c|}{ Pretest } & \multicolumn{2}{|c|}{ Postest } & \multicolumn{2}{|c|}{ Prueba t } & \multirow{2}{*}{$\begin{array}{c}\text { Tamaño del } \\
\text { efecto } \\
\text { d }\end{array}$} \\
\hline & M & DT & M & DT & $\mathrm{t}$ & $p$ & \\
\hline Fluidez & 5.22 & 5.08 & 18.26 & 9.23 & -7.39 & $.000^{\star \star \star}$ & $1.75^{\wedge \wedge \wedge}$ \\
\hline Originalidad & 9.52 & 7.88 & 18.59 & 10.25 & -4.73 & $.000^{\star * *}$ & $0.96^{\wedge \wedge \wedge}$ \\
\hline Elaboración & 17.85 & 16.31 & 41.44 & 15.12 & -7.08 & $.000^{\star * *}$ & $1.50^{\wedge \wedge \wedge}$ \\
\hline Títulos & 10.33 & 10.34 & 17.70 & 14.01 & -2.31 & $.000^{\star * *}$ & $0.59^{\wedge \wedge}$ \\
\hline Cierre & 6.26 & 5.14 & 25.48 & 8.78 & -11.13 & $.029 *$ & $2.67^{\wedge M \wedge}$ \\
\hline Bonus & 6.26 & 8.02 & 33.19 & 13.35 & -9.10 & $.000^{* * *}$ & $2.44^{\wedge \wedge \wedge}$ \\
\hline Total & 56.26 & 45.37 & 154.67 & 60.77 & -8.20 & $.000^{\star * *}$ & $1.83^{\wedge \wedge \wedge}$ \\
\hline
\end{tabular}

Nota: Estadísticamente significativas: ${ }^{\star} p<.05{ }^{*} p<.01 * \star * p<.001$

$\wedge$ Tamaño del efecto pequeño: $d \leq .49^{\wedge}$ Tamaño del efecto moderado: $d=$ entre .50 y .79

$\wedge \wedge \wedge$ Tamaño del efecto grande: $d d \geq .80$

En cuanto a la significación estadística, se observa que se producían diferencias estadísticamente significativas $(p<.05)$, entre el pretest y el postest, a favor del segundo y, por tanto, el programa había tenido un efecto sobre el desarrollo de la creatividad en los participantes.

Si se tiene en cuenta la vía de la significación sustantiva, se obtiene un tamaño del efecto grande en todas las variables medidas; excepto en la variable "Títulos", en la que el efecto ha sido moderado. Estos resultados, señalan que hay evidencias empíricas suficientes para afirmar que la creatividad del grupo es sustantivamente mayor después de la intervención; y que el programa ha resultado eficaz.

Esta mejora de la creatividad, queda gráficamente plasmada en los siguientes ejemplos (ver Figuras 1, 2 y 3), en los que se pueden comparar las producciones realizadas en las actividades de test de Torrance et al. (1992), en el pretest y el postest de algunos de los participantes. 
Revista Internacional de Apoyo a la Inclusión, Logopedia, Sociedad y Multiculturalidad. Volumen 4, Número 3, Julio 2018, ISSN: 2387-0907, Dep. Legal: J-67-2015

http://riai.jimdo.com/

Figura 1: Ejemplo de la producción en la actividad "Construcción de dibujos" pre y postintervención

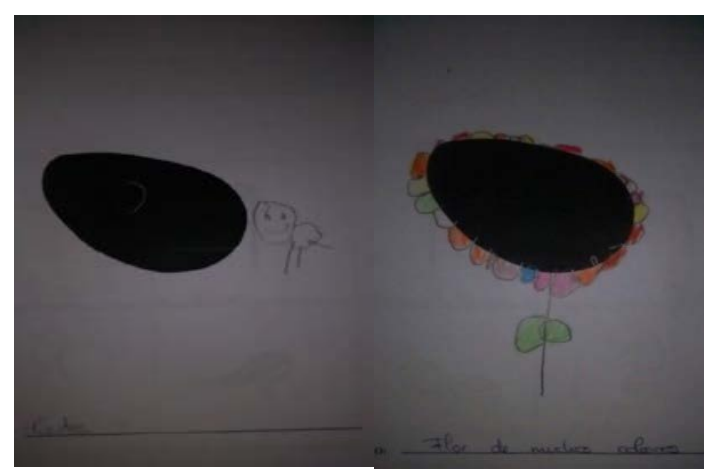

Fuente: elaboración propia (2018).

Figura 2: Ejemplo de la producción en la actividad "Terminación de dibujos" pre y postintervención

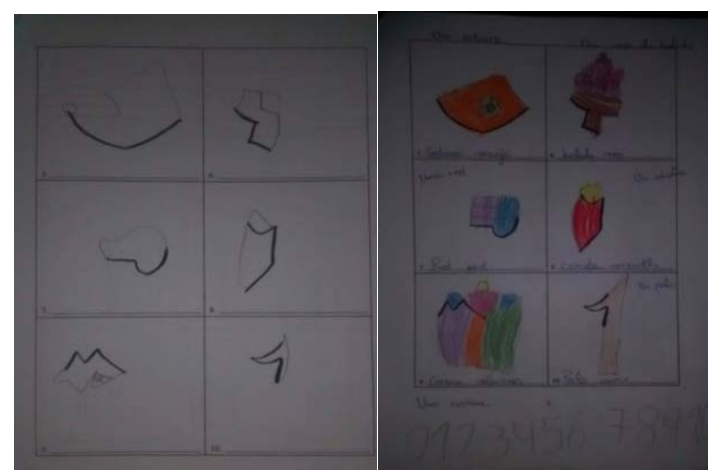

Fuente: elaboración propia (2018).

Figura 3: Ejemplo de la producción en la actividad "Líneas" pre y postintervención

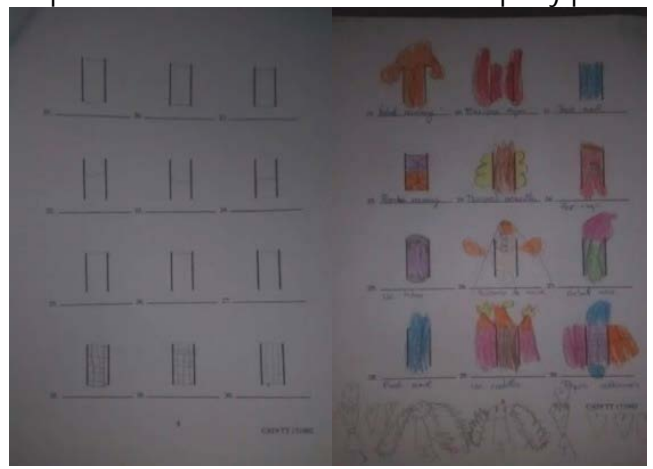

Fuente: elaboración propia (2018). 


\section{5.-Conclusiones.}

Este estudio muestra los efectos positivos del programa "¿Te atreves a crear?", sobre la competencia creativa de los niños de 4-5 años participantes. Partiendo de un enfoque integral, el programa pretende desarrollar la creatividad, atendiendo tanto al proceso creativo, como al resultado final, trabajando habilidades de pensamiento divergente, expresión plástica, musical y corporal, a través del juego y el aprendizaje cooperativo.

El programa ha demostrado, como los estudios previos (Alfonso-Benlliure et al., 2013; Antonietti, 2000; Castillo, 1998; Cliatt, Shaw y Sherwood, 1980; Dziedziewicz et al., 2013; Garaigordobil y Berrueco, 2011; Mohanty y Hejmadi, 1992; Prieto, López, Bermejo, Renzulli y Castejón, 2002; Useem y Downie, 1976), que es posible estimular las habilidades creativas desde la edad más temprana, y que es una herramienta útil para ser utilizada por los maestros de Educación Infantil.

Durante el proceso de realización de las sesiones, se han podido observar cambios gradualmente positivos. En un principio las actividades planteadas eran simples, y aun así, a muchos de los alumnos les costaba desenvolverse de manera autónoma, pidiendo ayuda continuamente o participando levemente en las actividades. Poco a poco, esto ha ido cambiando, habiendo un gran progreso en muchos de estos niños, que eran más reacios a expresarse o realizar las actividades de manera más activa. Se puede decir que todos ellos han participado, han aprendido y han descubierto nuevas formas de expresarse. Diariamente, han sido ellos los que querían seguir jugando y realizar nuevas obras, juegos, pintar con las manos, etc. La evolución de su competencia creativa ha sido muy positiva, avanzando en la mayoría de los casos; en otros, se ha mantenido más estable, aunque teniendo progresión positiva en algunos de los aspectos evaluados.

Por otro lado, haciendo balance sobre el estudio, se pueden hacer propuestas de mejoras, como modificar ciertos materiales de algunos de los juegos, alargar algunas de las actividades dada la motivación mostrada por los niños, trabajar más con las curiosidades que a los niños les van surgiendo, etc.

Como limitaciones se puede resaltar que para comprobar la eficacia del programa hubiese sido deseable haber contado con un grupo control, pero al tratarse de un centro escolar de una sola línea no era posible. Aun así, los resultados ya marcan una considerable mejora después de la intervención.

Esto lleva a concluir que todo docente, pero muy especialmente, los de Educación Infantil, deben ser conscientes de la influencia positiva que, sobre el desarrollo posterior del niño, tienen las prácticas creativas durante la infancia (Alfonso-Benlliure et al., 2013), y no sólo ser consciente, si no tener la suficiente formación y competencia para potenciarla de la mejor manera en el aula. 


\section{6.-Referencias.}

Alfonso-Benlliure, V., Meléndez, J.C. y García-Ballesteros, M. (2013). Evaluation of a creativity intervention program for preschoolers. Thinking Skills and Creativity, 10, 112-120. http://dx.doi.org/10.1016/i.tsc.2013.07.00

Beltrán, C.Y., Garzón, D.M. y Burgos, N.C. (2016). Incidencia del fortalecimiento del pensamiento divergente en la creatividad de los niños. Infancia Imágenes, 15(1), 103-118.

Dziedziewicz, D., Oledzka, D. y Karwowski, M. (2013). Developing 4- to 6-year-old children's figural creativity using a doodle-book program. Thinking Skills and Creativity, 9, 85-95. http://dx.doi.org/10.1016/j.tsc.2012.09.004

Garaigordobil, M., y Berrueco, L. (2011). Effects of a play program on creative thinking of preschool children. The Spanish Journal of Psychology, 14, 608618.

López Megías, L. y Fernández-Castillo, A. (2018). Creatividad en la Infancia Temprana. Análisis Cualitativo en un Contexto Educativo. ReiDoCrea, 7, 4354.

Ma, H.H. (2006). A synthetic analysis of the effectiveness of single components and packages in creativity training programs. Creativity Research Journal, 18, 435-446.

Prieto, M.D., López, O., Bermejo, M.R., Renzulli, J. y Castejón, J.L. (2002). Evaluación de un programa de desarrollo de la creatividad. Psicothema, 1, 410-414.

Scott, G., Leritz, L.E., y Mumford, M.D. (2004a). Types of creativity training: Approaches and their effectiveness. Journal of Creative Behavior, 38, 149179.

Scott, G., Leritz, L.E., y Mumford, M.D. (2004b). The Effectiveness of Creativity Training: A Quantitative Review. Creativity Research Journal, 16(4), 361-388.

Torrance, E.P., Ball, O.E. y Safter, H.T. (1992). Torrance Test of Creative Thinking. Streamlined Scoring Guide Figural A and B. Bensenville. II.: Scholastic Testing Service, Inc. 